\title{
Drug prescribing pattern in elderly patients in a teaching hospital
}

\author{
Veena D.R ${ }^{1}$, Padma L ${ }^{2}$, Sapna Patil ${ }^{3}$ \\ 1, 2, 3(Department of Pharmacology, Dr. B. R. Ambedkar Medical College \&Hospital, Bangalore, India)
}

\begin{abstract}
Objective: The present study was undertaken to study prescribing pattern of various drugs in elderly patients \& also to evaluate inappropriate prescribing with the help of Beers criteria. Material \& methods: The study was conducted in Dr.B.R.Ambedkar Medical College \& Hospital from June 2012 to August 2012. A total of 106 case records of inpatients >/65years in medicine wards were reviewed. Relevant information was recorded in a structured proforma \& data was evaluated. Results: Most of the cases were from respiratory system (35.84\%), followed by cardiovascular system (14.15\%). The most commonly prescribed drugs were antimicrobials (16.94\%). Polypharmacy was observed in 94(88.67\%) patients. According to Beers criteria, $23(21.69 \%)$ patients were prescribed inappropriately \& all these were drugs to be generally avoided in older adults. Conclusion: This study has shown the patterns of diseases prevalent in geriatric patients, drug use among them and also suggests that drugs to be avoided in elderly are among the most frequently inappropriately prescribed drugs. Prevalence of polypharmacy was high which is usually unavoidable in the elderly.
\end{abstract}

Keywords: Beers criteria, elderly, polypharmacy

\section{Introduction}

The elderly population is increasing rapidly worldwide. Interindividual differences in age related pharmacokinetic \& pharmacodynamic changes as well as co-morbid conditions have to be considered while prescribing medicines in elderly population ${ }^{1}$. Changes in the pharmacokinetic profiles of drugs occur in the elderly because of reduced body water, reduced renal and hepatic function and increased body fat. Multiple drug use \& polypharmacy is highly prevalent in elderly exposing them to drug interactions\& increased cost of therapy $y^{2,3}$. Physiological aging \& Alzheimer's disease also affects compliance. Hence this study was undertaken to identify the pattern of inappropriate use of medicines in this population which may help to prevent adverse drug reactions.

\section{Objectives}

a. To study prescribing pattern of various drugs in elderly patients.

b. To evaluate inappropriate prescribing with the help of Beers criteria.

\section{Materials \& Methods}

A prospective observational study was undertaken for 3 months duration from June 2012 to August 2012 in Dr. B.R. Ambedkar Medical College \& Hospital, Bangalore, India. The study protocol was approved by the Institutional Ethics Committee. 106 patients of geriatric age group (>/65 years) were included in the study. Data was obtained from Medical Records Section about the inpatients admitted to medicine wards. Data was collected in a structured proforma, which included patient's demographic details, inpatient registration number, diagnosis \& complete prescription.

\section{Results}

106 case records were analyzed during the study period. Most of the patients were in the age group of 65-70yrs (79.24\%).The study population comprised of 55.66\% males. Maximum of these cases were from respiratory system $(35.84 \%)$, followed by cardiovascular system (14.15\%)- Fig 1. COPD \& complications due to hypertension were the most common reasons for hospital admission. Polypharmacy was observed in $88.67 \%$ cases. 5-8 drugs were prescribed for most patients $(46.22 \%)$, followed by $>8$ drugs $(42.45 \%)$-Fig 2 . Total number of FDCs prescribed was 79 (14.87\%).

Antimicrobial drugs $(16.94 \%$ ) followed by GI protective agents $(13.93 \%) \&$ antihypertensives $(9.98 \%)$ were the most commonly prescribed medications (TABLE1).

Based on Beers criteria, 23 (21.69\%) patients received potentially inappropriate prescription of at least one drug \& all these belong to category A(TABLE 2). A total of 531 formulations were prescribed out of which $23(4.33 \%)$ were prescribed inappropriately. 
Drug prescribing pattern in elderly patients in a teaching hospital

The most commonly prescribed group of antihypertensive drugs were diuretics (41.5\%), followed by calcium channel blockers (37.73\%).(TABLE 3).
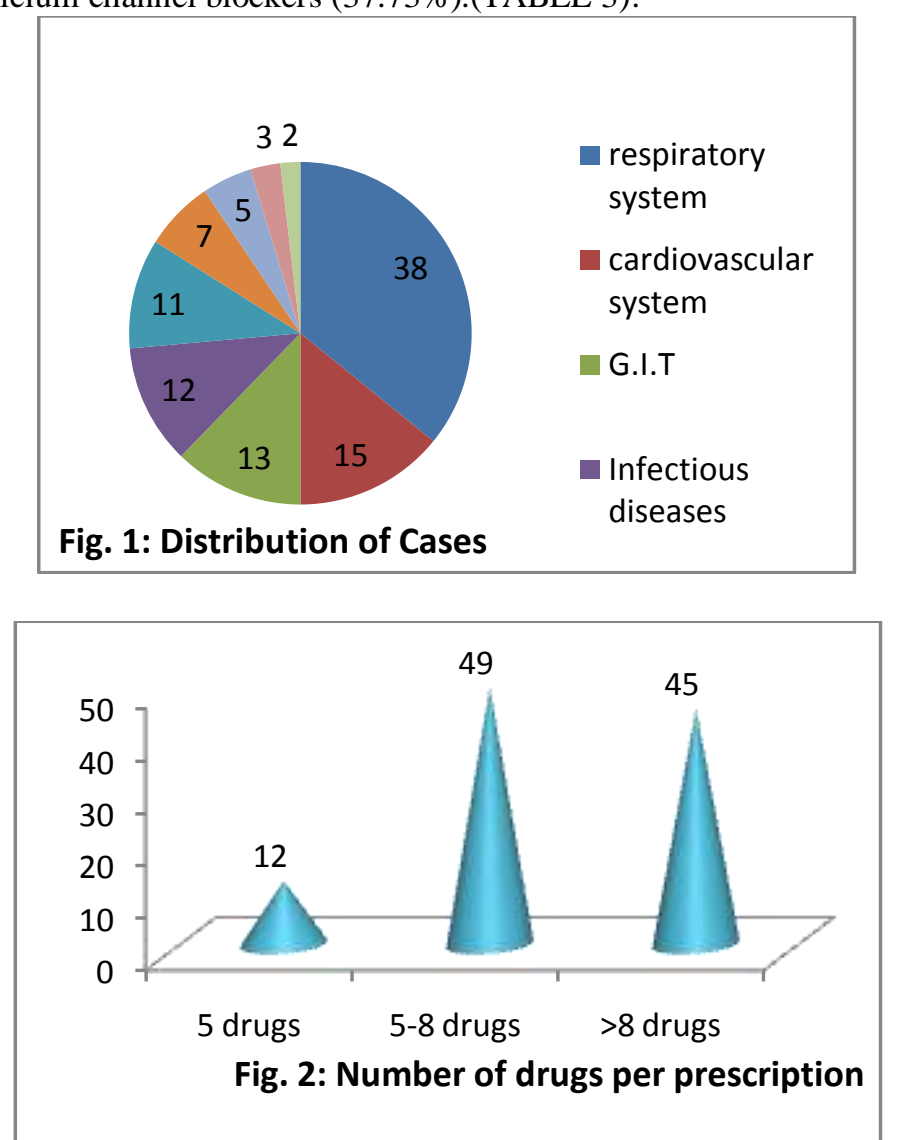

TABLE 1: Category of drugs prescribed

\begin{tabular}{|l|l|}
\hline Category of drugs & Number of drugs (\%) \\
\hline Drugs acting on Respiratory System & $106(19.96 \%)$ \\
\hline Antimicrobial drugs & $90(16.94)$ \\
\hline Drugs acting on Gastrointestinal System & $88(16.57)$ \\
\hline Cardiovascular drugs & $68(12.8)$ \\
\hline Drugs acting on Endocrine system & $49(9.22)$ \\
\hline Drugs acting on Haematological system & $46(8.66)$ \\
\hline Analgesics \& anti-inflammatory drugs & $41(7.72)$ \\
\hline Drugs acting on Central Nervous System & $21(3.95)$ \\
\hline Vitamins, minerals \& dietary supplements & $15(2.82)$ \\
\hline Others* & $07(1.31)$ \\
\hline TOTAL & $\mathbf{5 3 1}$ \\
\hline
\end{tabular}

*- Antihistaminics, skeletal muscle relaxants

TABLE 2: Frequency of use of potentially inappropriate medicines in elderly based on Beers criteria

\begin{tabular}{|l|l|l|}
\hline Category & Name of Drugs & Total=23 \\
\hline A & Generally to be avoided in older adults & \\
\hline & Hyoscine & 06 \\
\hline & Alprazolam & 05 \\
\hline & Amitryptilline & 04 \\
\hline & Chlorpheniramine & 03 \\
\hline & Diazepam & 02 \\
\hline B & Glibenclamide & 02 \\
\hline C & Dicyclomine & 01 \\
\hline & Drugs that exceed maximum recommended daily dose & Nil \\
\hline & $\begin{array}{l}\text { To be avoided in combination with specific co- } \\
\text { morbidity }\end{array}$ & Nil \\
\hline
\end{tabular}


Drug prescribing pattern in elderly patients in a teaching hospital

TABLE 3: Quantitative distribution of prescribed Antihypertensive drugs

\begin{tabular}{|l|l|}
\hline Antihypertensives & Number (\%) \\
\hline Diuretics & $22(41.5)$ \\
\hline Calcium channel blockers & $20(37.73)$ \\
\hline ACE inhibitors & $08(15.09)$ \\
\hline Beta blockers & $03(5.66)$ \\
\hline Total & $\mathbf{5 3}$ \\
\hline
\end{tabular}

\section{Discussion}

Inappropriate prescribing can be defined as prescribing medications outside the bounds of accepted medical standards ${ }^{4}$.Maximum of diagnosed cases were from respiratory system (35.84\%), followed by cardiovascular system $(14.15 \%)$. COPD \& complications due to hypertension were the most common reasons for hospital admission. In a previous study among the elderly at Kathmandu, respiratory and cardiovascular diseases were shown to be the predominant reasons for admission ${ }^{5}$.

In this study, a total of 531 drug formulations were prescribed to 106 patients for different diseases. Polypharmacy was observed in $88.67 \%$ cases. 5-8 drugs were prescribed for most patients (46.22\%), followed by $>8$ drugs $(42.45 \%)$. This is similar to another study in Karnataka where average number of medications was $9.4^{6}$. Of these drug formulations, $14.87 \%$ were fixed drug dose combinations. Polyphrmacy is increasing because of not only co-morbid conditions but also due to increased awareness about drugs, literate elders or care takers and pressure on the physicians to prescribe a drug for each symptom.

Antimicrobial drugs (16.94\%) followed by GI protective agents (13.93\%) \& anti-hypertensives ( $9.98 \%$ ) were the most commonly prescribed medications .

Beers criteria are very frequently used method for evaluating appropriateness of prescribing in elderly. It was developed in 1999 \& recently updated in 2012.In the present study, according to Beers criteria, it was revealed that $4.33 \%$ of total drugs prescribed were inappropriate as compared to $4.1 \%$ in an earlier study conducted in south India ${ }^{6} .23(21.69 \%)$ patients received potentially inappropriate prescription of at least one drug $\&$ all these belong to category A which includes drugs to be avoided in older adults.

Anticholinergics, antihistaminics, long acting sulfonylureas \& benzodiazepines were prescribed to 23 patients. Long acting benzodiazepines (diazepam) can cause hangover effects, concomitant increase in falls. Hence short acting benzodiazepines like oxazepam is preferred. Among geriatric patients, dizziness, postural hypotension, constipation, delayed micturition are found commonly with tricyclic antidepressants. Counselling elderly patients and their family members may help in improving their mood rather than drug therapy for depression. Long acting sulphonamides like glibenclamide may cause hypoglycaemia and hence glimepiride is preferred which retains the ability of glucose dependent inhibition of insulin secretion during hypoglycaemia.

However, these findings is not significantly different from that found in a study from Japan ${ }^{7}$ Ahmedabad $^{8} \&$ Gujrat $^{9}$ which observed use of at least one inappropriate medicine in $21.1 \%, 23.58 \%$ $\& 27.25 \%$ prescriptions respectively. This suggests that drugs 'to be avoided in elderly' are among the most frequently inappropriately prescribed drugs.

The most commonly prescribed group of antihypertensive drugs were diuretics( $41.5 \%)$, followed by calcium channel blockers $(37.73 \%)$. Thiazide diuretics are recognized as the cornerstone of antihypertensive therapy because of their extensive track record in preventing stroke and cardiovascular events and their low $\operatorname{cost}^{10}$. Dihydropyridine CCBs reduce stroke by $10 \%$ compared with other active therapies ${ }^{11}$. CCBs are acceptable alternatives to diuretics for first-line treatment of hypertension in the elderly and may offer advantages in some patient groups, eg, those with metabolic syndrome.

\section{Conclusion}

This study has shown the patterns of diseases prevalent in geriatric patients, drug use among them and also suggests that drugs to be avoided in elderly are among the most frequent inappropriately prescribed drugs. Prevalence of polypharmacy was high $\&$ is usually unavoidable in the elderly. This indicates that there is a need for multidisciplinary, multifaceted \& multisector approach which may improve drug safety \& adherence in the elderly. 
Limitations of the study include small sample size \& restriction to one speciality. Larger studies involving elderly patients in various departments are necessary to realise the impact of this serious problem $\&$ to make prescriptions more rational in this group of population.

It also emphasizes the need for creating more awareness among the general practitioners \& clinicians on this important public health issue by conducting more Continued Medical Education programmes on drug therapy in elderly. In view of academic interest this topic can be included in medical text books in an effort to improve the quality of health care.

\section{References}

[1] McLean AJ, Le Couteur DG, Aging biology and geriatric clinical pharmacology, Pharmacol Rev,56 ,2004,163-84.

[2] Jorgensen T, Johansson S, Kennerfalk A, Wallander MA, et al, Prescription drug use, diagnoses, and healthcare utilization among the elderly, Ann Pharmacother ,35,2001,1004-9.

[3] Kennerfalk A, Ruigomez A, Wallander MA, Wilhelmsen L, Johansson S, Geriatric drug therapy and healthcare utilization in the United kingdom, Ann Pharmacother,36, 2002, 797-803.

[4] Spinewine A, Schmader KE,Barber N,etal, Appropriate prescribing in elderly people.How well can it be measured \& optimised? Lancet ,370, 2007,173-84.

[5] Lohani SP, Thapa P, Aryal UR, Satyal KR, Polypharmacy and geriatric patients: patterns of prescribing in the Tribhuvan University Teaching Hospital in Nepal. J Nepal Health Res Counc ,4,2006,1-4.

[6] Harugeri A, Joseph J, Parthasarathi G, Ramesh M, Guido S, Potentially inappropriate medication use in elderly patients: A study of prevalence and predictors in two teaching hospitals. J Postgrad Med ,56, 2010, 186-91.

[7] Niwata S, Yamada Y, Ikegami N, Prevalence of inappropriate medication using Beers criteria in Japanese long term care facilities. BMC Geriatr, 6, $2006,1$.

[8] Zaveri HG, Mansuri SM, Patel VJ, Use of potentially inappropriate medicines in elderly: A prospective study in medicine outpatient department of a tertiary care teaching hospital.Indian J Pharmacolgy,42(2),2010,95-98.

[9] Shah RB, Gajjar BM, Desai SV, Evaluation of the appropriateness of prescribing in geriatric patients using Beers criteria and Phadke's criteria and comparison thereof, J Pharmacol Pharmacother,2(4), 2011,248-52.

[10] Chobanian AV, Bakris GL, Black HR, et al, Seventh Report of the Joint National Committee on Prevention, Detection, Evaluation, and Treatment of High Blood Pressure. Hypertension. 2003,42,1206-1252.

[11] Angeli F, Verdecchia P, Reboldi GP, et al, Calcium channel blockade to prevent stroke in hypertension: a meta-analysis of 13 studies with 103,793 subjects. Am J Hypertens, 17, 2004,817-822. 